University of South Carolina

Scholar Commons

\title{
"In the Beginning, It Was Little Whispers...Now, We're Almost a Roar": Conceptualizing a Model for Community and Self in LGBTQ+ Health Information Practices
}

\author{
Vanessa Kitzie \\ University of South Carolina, kitzie@mailbox.sc.edu \\ Travis L. Wagner \\ University Of South Carolina, wagnertl@email.sc.edu \\ Alexander N. Vera \\ University Of South Carolina, veraan@email.sc.edu
}

Follow this and additional works at: https://scholarcommons.sc.edu/libsci_facpub

Part of the Lesbian, Gay, Bisexual, and Transgender Studies Commons, and the Library and Information Science Commons

Publication Info

Preprint version iConference Sustainable Digital Communities, 2020, pages 1-18.

(c) The Authors, 2019

This Conference Proceeding is brought to you by the Information Science, School of at Scholar Commons. It has been accepted for inclusion in Faculty Publications by an authorized administrator of Scholar Commons. For more information, please contact digres@mailbox.sc.edu. 


\title{
"In the beginning, it was little whispers...now, we're almost a roar": Conceptualizing a model for community and self in LGBTQ+ health information practices
}

\author{
Vanessa L. Kitzie[0000-0002-6499-9584], Travis L. Wagner[0000-0002-6000-157X], and A. Nick \\ Vera[0000-0001-5715-7293] \\ University of South Carolina, Columbia, South Carolina, USA \\ kitzie@mailbox.sc.edu
}

\begin{abstract}
Although LGBTQ+ populations experience significant health challenges, little research exists that investigates their health from an informational perspective. Our study addresses this gap by exploring the health information practices of LGBTQ+ communities in South Carolina, focusing on how sociocultural context shapes these practices. Thirty semi-structured interviews with South Carolina LGBTQ+ community leaders analyzed using open qualitative coding informed the development of a conceptual framework describing their information practices. Findings show that participants engaged in two broad types of practices - protective and defensive - as responses to risks and barriers experienced, which are in turn produced by social and structural factors. Findings advance information practices and marginalization approaches and offer ways for medical professionals to improve service to LGBTQ+ populations.
\end{abstract}

Keywords: Information Practices, LGBTQ+ Communities, Health Information.

\section{$1 \quad$ Introduction}

Lesbian, gay, bisexual, transgender, and queer $\left(\mathrm{LGBTQ}^{+}\right)$people face significant health challenges compared to their cisgender and heterosexual peers. These challenges are produced by social and structural factors, e.g., discrimination, resulting in mental and physical health disparities among this population [1-3]. Disparities can be specific to sub-groups under the broader LGBTQ+ umbrella and vary based on other intersecting, contextual, and identity-related aspects [1-2, 4-5]. A key factor contributing to these health challenges is informational, as LGBTQ+ people may experience difficulty learning about their healthcare needs, navigating the healthcare system, and addressing barriers to care [6-7]. Despite the significance of information in shaping LGBTQ+ health outcomes, few studies investigate LGBTQ+ health from an informational perspective. Further, existing work frames this phenomenon using deficit-based models, which disempower LGBTQ+ populations [8].

LGBTQ+ residents in the Southern U.S. states may experience enhanced health challenges, since these regions have more conservative views of sexuality and gender 
identity on average [9]. South Carolina (SC) is a predominantly rural Southern state with

$2.9 \%$ of the population identifying as LGBTQ+ [10]. LGBTQ+ residents experience more severe health challenges as compared to the national averages, including heightened economic instability, unemployment, and lack of health insurance [11]. While some quantitative work documents the health challenges of SC LGBTQ+ residents [e.g., 10,12] there exists an absence of qualitative research providing in-depth perspectives of how individuals navigate these challenges in their everyday lives.

This research addresses the above gaps by examining the health information practices of SC LGBTQ+ communities with a focus on how sociocultural context shapes these practices. By employing a social constructionist approach, the research envisions communities as engaging in information practices for personal and community empowerment in response to health challenges faced.

\subsection{Theoretical Orientations}

Information Practices Approach. Information behavior and practice are two umbrella concepts describing how people deal with information [13]. While their boundaries are blurry in practice, these concepts exhibit distinct epistemological and ontological understandings of information's social aspects [13-14]. The cognitivist information behavior approach frames information needs as drivers of seeking and use. This framing has positivist and behaviorist metatheoretical underpinnings [15], which can be wielded against specific populations to suggest that any behaviors not adhering to this limited view are indicative of a personal failing requiring correction from experts [16-18].

Information practices represent constructivist and constructionist perspectives, where social and structural factors shape people's relationships with information. Information practices research focuses on the everyday life contexts in which people interact with information [19] and has uncovered relevant practices beyond seeking, including sharing and use [14]. We adopt an information practices approach for this study as it addresses how broader social and structural factors differentiate the health issues experienced by LGBTQ+ people from their heterosexual and cisgender peers. This perspective aligns with population health [20] and resilience approaches [21] addressing social and structural determinants of health.

Information Marginalization Theory. Information marginalization theory adopts a sociocultural lens to examine information inequality, wherein specific populations experience enhanced challenges in dealing with information [22]. Information poverty theory [23] and a constructivist grounded theory analysis [22] inform information marginalization theory. It has three components: 1) information marginalization factors, 2) individual-level behaviors, and 3) community-level practices. Per the theory, individual-level behaviors and community-level practices respond to information marginalization factors, often to defend/protect (used synonymously) against them [22]. This theory informs our information practices approach by identifying defensive/protective information practices as a typical response to social and structural 
inequalities and addressing how these practices operate at both individual and community levels of analysis.

\section{$2 \quad$ Literature Review}

Library and Information Science (LIS) scholars have applied an information practices approach to marginalized groups based on its strengths in attending to sociocultural context. Research highlights nuances and differences to traditional information seeking models and theories in ways that can potentially disrupt or re-situate them [14, 19, 23]. Several LIS studies offer insight into the unique relationship between information practices and information marginalization.

Studies examining information marginalization across a variety of populations demonstrate that despite engaging in certain practices that appear less "mainstream" [24] participants are knowledgeable and agentic in their information interactions. Specifically, they are aware of social and structural barriers to information and engage in information practices as measured responses. Examples include immigrants using tactical information like sharing between community elders and wandering to learn about a city's layout [25]; young parents sharing information via mobile technologies in lieu of "traditional" sources for health information [26]; and parents of individuals with disabilities designating a point person to gather information for the community based on limited access to health and wellbeing resources [22].

Few LIS studies focus on the information practices of LGBTQ+ populations. Existing work highlights the importance of community contexts, the need to manage the visibility of information practices, and community mistrust of outside information sources. Communities work together to maintain the trustworthiness of health information by establishing social capital and engaging in networked bonding and bridging to share information [27]. In other cases, the anonymity of online spaces provides a way for communities to safely seek information [28]. Other work considers the consumption of established LGBTQ+ information resources (i.e., media) within the community as purposeful "satisficing" based on the collective perception that limited representation is better than none [29]. Some research studies outside of LIS apply an informational focus to LGBTQ+ health; for instance, prior research identified how negative past experiences with health practitioners shaped LGBTQ+ patients' sharing and seeking practices, specifically disclosure management and avoidance [30-31]. While these studies and related work begin a necessary dialogue around LGBTQ+ information practices, there remain considerable gaps in the literature.

Those able to participate in institutional dialog about their identities and needs tend to come from privileged groups. As a result, studies examining LGBTQ+ identity and popular discourses at large center the needs and desires of cisgender, gay, white men and lesbians of relative wealth. This identity normalizes and dictates an LGBTQ+ agenda exclusionary of non-white gay cisgender men [32]; transgender and gender nonconforming persons of color [33]; and non-Western LGBTQ+ individuals [34]. This lack of representation is evident in the studies overviewed [27-29], noting the overt presence of white, well-educated participants as a limitation. Similarly, much of the 
research focusing on $\mathrm{LGBTQ}+$ individuals predominantly looked at millennial populations [28-29]. Further, [27-28]'s research recruited participants in mostly urban areas, reifying metronormativity narratives, which singularize the experiences of LGBTQ+ individuals inhabiting metropolitan spaces as universalized narratives [35].

Research reviewed describes how social and structural factors shape people's information practices. Additionally, those who are socially and structurally marginalized face unique challenges when dealing with information. How they address these challenges results in a series of nuanced practices that differ from those who do not experience such alterity. This study responds to a critical lack of information behavior and practices literature on LGBTQ+ individuals and focuses on their health information challenges, an unexplored area among this population. Further, it attends to some of the above sampling gaps by recruiting participants with diverse sexualities, gender identities, ages, races/ethnicities, and education levels from SC. The study addresses the following research question: How does sociocultural context shape the health information practices of SC LGBTQ+ communities?

\section{$3 \quad$ Methods}

This qualitative study was approved by the University of South Carolina Institutional Review Board (IRB) for expedited review (Pro0008587). We recruited 30 SC LGBTQ+ community leaders to participate in individual, semi-structured interviews between January - August 2019. This sample size is appropriate to study's goal of advancing theory rather than generating a priori codes [36-37]. Leaders serve as vital stakeholders within their communities and have a valuable, macro-level vantage point from which to examine them. Our definition of "community" employs three criteria: 1) geography, participants reside or perform a majority of community work in SC, 2) social interaction, members engage in shared activities, and 3) ties, members are connected via shared LGBTQ+ identities [38]. Additional criteria specified that participants were 13 years or older. We included youth in our sample, given the many examples of dynamic youth LGBTQ+ leaders [39-40]. Since some youth wished to participate without outing themselves to a parent or guardian, we obtained an IRB waiver of informed consent and instead collected youth assent [see also 30].

We used purposive sampling to collect names and contact information for over 100 visible LGBTQ+ and affinity organizations (e.g., social justice organizations, Unitarian Universalist Churches) in SC. We then contacted these organizations via email, asking them to nominate a leader to participate. Additional snowball sampling occurred at the end of each interview when we asked leaders to recommend other participants. Since data collection and analysis were iterative, we used theoretical sampling to identify perspectives that could flesh out or potentially contradict emergent findings, which led us to engage in additional recruitment via social media.

The semi-structured interview protocol addressed the following topics: a) participant's involvement with the community, b) participant and members identities, c) community health questions and concerns, and d) how the community addresses health questions and concerns. We structured questions from topic d) based on [14]'s 
model of information practices, which divides them into seeking, sharing, and use. We also added creating as another relevant practice uncovered in research on teens and LGBTQ+ individuals [28-29, 41]. Following interviews, we asked participants to elicit topics c) and d) further using information worlds mapping, a visual arts elicitation method [42]. We pre-tested the protocol and mapping exercise with three SC nonLGBTQ+ community leaders before participant interviews.

The mean and median interview duration was 78 minutes, and participants received a $\$ 50$ cash incentive. Interviews were face-to-face at a public location of the participant's choosing except two interviews conducted over the phone. One participant was disabled and could not meet in-person, while another youth wanted to participate, but feared rejection from her family if they discovered her participation. In these two cases, participants did not engage in the mapping exercise. One or two paper authors attended each interview and took detailed field notes post-interview. All interviews were audiorecorded and transcribed verbatim.

Data sources were interview transcripts, field notes, and information worlds maps. Per [42]'s earlier work, we viewed the maps as supplemental to transcripts when performing analysis. We analyzed data using an open qualitative coding process borrowed from, but not identical to, grounded theory [43]. First, we individually handcoded three transcripts using initial, process, and in vivo coding [37]. We then met to compare and discuss codes, generating a preliminary codebook. We applied this codebook to four additional transcripts, meeting six times to discuss emergent codes and compare them to existing ones [44]. After discussing and resolving coding discrepancies, we divided and independently coded the remaining transcripts. Then, we deployed focused and axial coding to describe conceptual categories and the relationships between them. We engaged in member-checking, sending participants their transcripts and fieldnotes to request removal of potentially identifying contextual information and comment on how well they reflected lived experiences [45].

\section{$4 \quad$ Findings}

\subsection{Participant Demographics}

The following Figures 1-2 and Table 1 display participant demographic data. The majority of participants were young adults (18-35) and middle-aged (35-54), while their races/ethnicities reflected the broader state-level demographics for LGBTQ+ people [10]. Participant education levels varied but trended toward those with some degree of higher education. While the geographic distribution of participants spread across the state, more leaders resided in the Upstate and Midlands regions where LGBTQ+ and affinity organizations are prevalent and active. Fewer participants in the Coastal Lowcountry and Pee Dee regions likely reflects their smaller populations and less visible organizations. One exception was Charleston, which our sample underrepresented. While the majority of participants identified as lesbian and gay, they employed many other labels to describe their sexualities and gender identities. These 
labels denote the inability of umbrella labels like LGBTQ+ to capture the multiplicity and fluidity of participant identities.

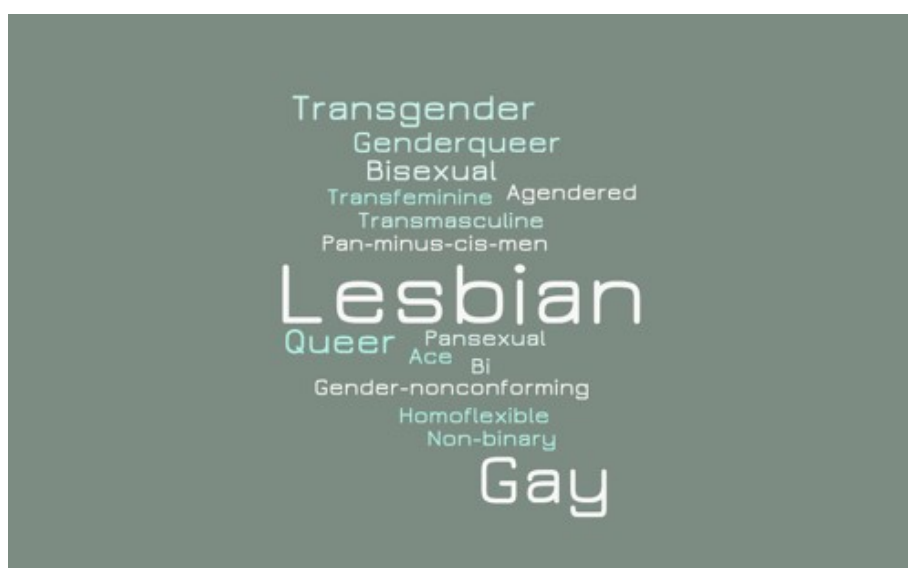

Fig. 1. Word cloud displaying participant gender and sexuality labels.

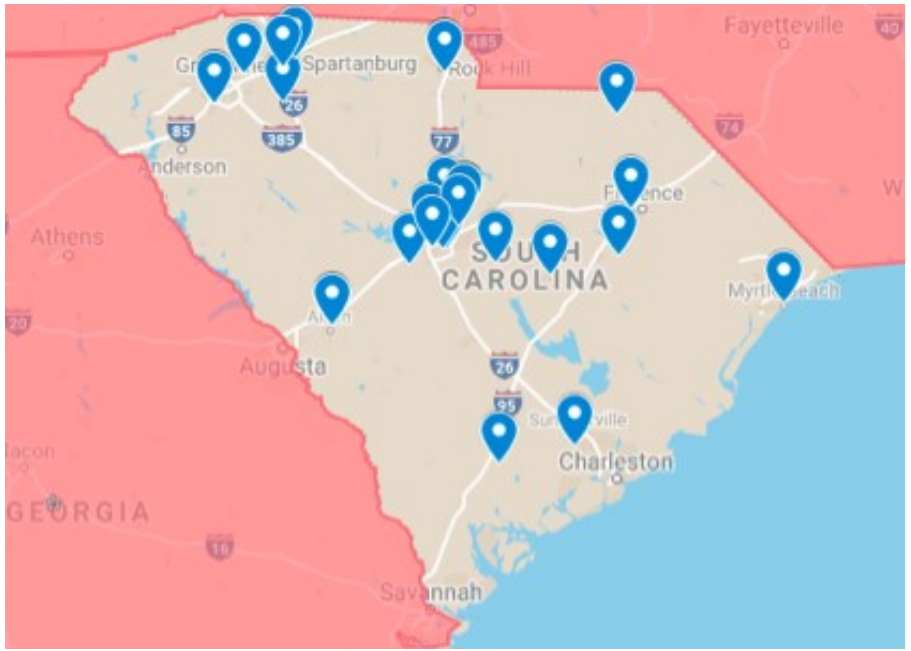

Fig. 2. Map of participant locations. 
Table 1. Participant age ranges, race \& ethnicity, and education.

\begin{tabular}{lc|lc|lc}
\hline Age & N & Race \& Ethnicity & N & Education & N \\
\hline $18-25$ & 11 & White & 18 & Some college credit & 7 \\
$35-54$ & 7 & Black & 7 & Master's degree & 6 \\
$55-64$ & 4 & Black, White & 2 & Associate degree & 5 \\
$13-17$ & 4 & Black, Afro-Caribbean & 1 & Bachelor's degree & 5 \\
$26-34$ & 3 & Aboriginal, Arab/West Asian, Black, White & 1 & In high school & 2 \\
$65+$ & 1 & Black, White, Egyptian & 1 & In middle school & 2 \\
& & & & High school diploma & 1 \\
& & & Doctoral degree & 1 \\
& & & GED or alt. credential & 1 \\
\hline
\end{tabular}

4.2 Conceptual Model of Participants' Health Information Practices

Data analysis and the study's theoretical orientation [14, 22-23] informed the development of a conceptual model describing participants' health information practices (Figure 3). The model's first component, contextual conditions, represent the underlying circumstances shaping how participants interact with health information. These conditions are broad, encompassing structural dimensions such as identity, geography, and politics. Gender identity and sexuality are overarching contextual conditions for this study and intersect with other conditions to produce qualitatively distinct health information practices.

Contextual conditions produce two broad types of social and structural inequities: barriers and risks. Barriers are significant obstacles experienced in a specific spacetime arrangement that constrain individuals and communities from achieving desired informational outcomes. Examples of barriers identified by data analysis included family, law, and religion. Unlike barriers, which are actualized, risks represent perceived exposure to danger, harm, or loss that might result from engaging in specific information practices. Risks are often more specific than barriers, since barriers operate at the sociocultural level, whereas risks are more immediate and individualized. Examples of risks uncovered by data analysis were physical violence, being kicked out, and fear of the unknown. Barriers and risks are co-constitutive, with barriers commonly experienced by some rendered as potential risks to others and vice versa. Participants are not disempowered or lack agency because they face barriers and risks; instead, they respond to them to achieve particular informational outcomes. 


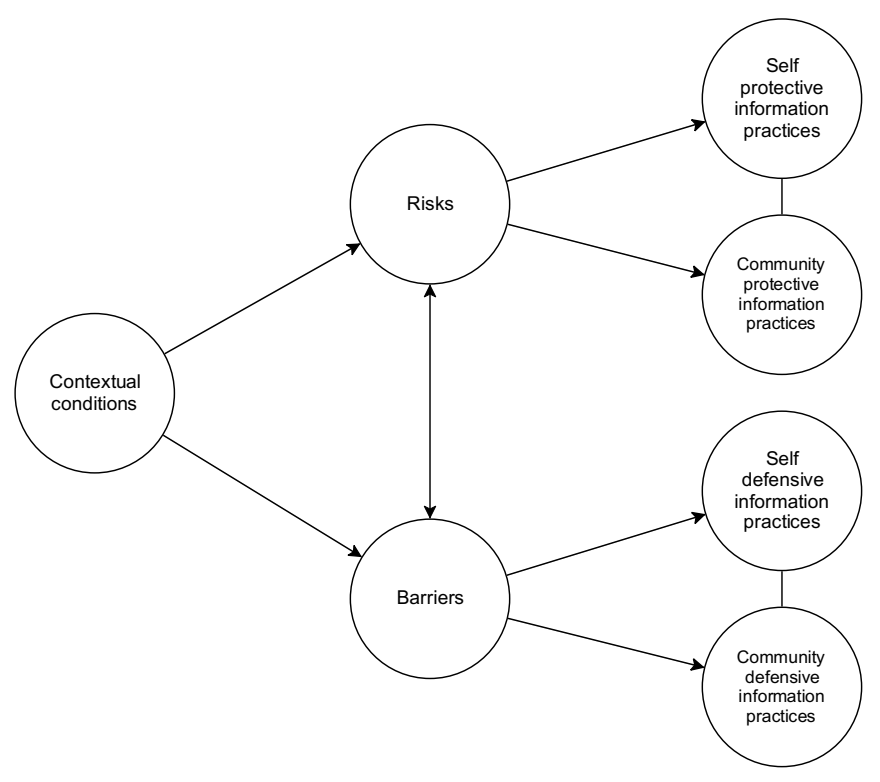

Fig. 3. Conceptual model describing participants' health information practices.

Barriers and risks produce two distinct types of information practices: defensive and protective. Defensive information practices are instances where individuals or communities create, seek, share, and use information to defend against a barrier or barriers. These practices are reactive; individuals and communities deal with information as a direct response to the barrier(s). Individuals and communities engage in protective information practices to guard against a perceived risk or risks. These practices are proactive; while the risk did not yet occur, it has the potential to become actualized. Because barriers and risks are co-constitutive, defensive and protective information practices can co-occur. Both types of information practices can be divided further at the self and community levels. Although community served as the unit of analysis for this work, in interviews, participants discussed health issues experienced not only by their communities on a collective level but also by individuals within their communities and themselves, hence this division.

\subsection{Illustrating the Conceptual Model with Participant Narratives}

The following section contextualizes the above model using thick description [46] from four participant accounts. We selected these narratives to illustrate variety in the "paths" taken by participants across the model. Importantly, our use of contextual conditions only reflects those necessary to the narrative. The absence of other contextual conditions is not to devalue their importance but to protect participant confidentiality. 
To maintain confidentiality further, we refer to participants using self-selected pseudonyms. We also refer to participants using their pronouns; when participants provided more than one set of pronouns, we vary them accordingly.

Narrative 1: Jake Hartwell. Jake Hartwell recalled his experience as a social worker in the level one trauma center of a hospital. During this period, he would sometimes have LGBTQ+ patients ask him health questions, such as "about certain sexual practices." While Jake could potentially share information based on his experience as a gay man, he did not want to be "too informative" and disclose this identity. Jake felt it was inappropriate to out himself to do this information sharing given his ostensible role as a neutral and unbiased party in the field of social work: "I don't want to necessarily be like, 'Well, this is what I do, and this is how I do it,' just because that's not really appropriate." Instead, Jake provided his patients with information he thought they would need as LGBTQ+ individuals without disclosing his identity: "I want my patients to be able to see me as a blank canvas because if I tell them I identify this way, or I have this value, that might be something for them that might be harmful."

Several contextual conditions shape Jake's information practices. While likely true of multiple participants, Jake made it clear during member-checking that all of his intersecting identities (not solely the ones we initially highlighted) inform his practitioner role. Salient areas of Jake's identity include being bisexual, a person of color, young adult, first generation graduate student, and person of faith. While these identities shape Jake's social work practice by motivating and encouraging his work, they are not viewed by Jake to be appropriate to share with patients because they are not neutral. Key barriers that may shape Jake's decision to not share his personal experiences are homophobia and transphobia. These barriers can lead to the additional job-related risks of appearing unprofessional and doing harm by being "off-putting" to patients. To respond to these conditions, barriers, and risks, Jake engages in several information practices. First, he assesses his areas of expertise as a social worker, bisexual, a person of color, young adult, first generation graduate student, and person of faith to inform how and what information he conveys to his LGBTQ+ patients. We argue that such assessment constitutes a form of community protective information use, in which Jake protects against appearing unprofessional and doing harm by determining which parts of his experience and expertise rooted in these experiences are relevant to share and how to share them. In turn, Jake chooses to avoid sharing these experiences and expertise based on his intersecting identities with the understanding that this avoidance facilitates access to the very people whom he sees as his community. These people have LGBTQ+ identities, but also other marginalized identities, such as people from broken families or minority racial groups. Jake refers to this community as "the island of misfit toys," a descriptor illustrating the homophobia and transphobia barriers (among other, intersecting ones including racism) this community faces. Jake operates within these barriers to provide non-discriminatory care, even if he is doing so from a performed vantage point of neutrality. We regard this choice as self-defensive information sharing since Jake works to defend himself against barriers that could call into question his professionalism and the role of social work more broadly. We further 
categorize this practice as disclosure management and envision Jake as intentionally choosing not to disclose his identity to maintain his professional aspirations, which are to help his patients, including his community, with their health questions and concerns.

Narrative 2: Ben. Ben is a high school student who defined his community as comprised of other LGBTQ+ students: "So we only have four gay people at school and so- actually, no, we have [NAME]. [...] Okay, we have him. And yeah, it's only us." At school, Ben described meeting with community members outside on school grounds "near where we put our garden" against "a brick wall." Sometimes during these meetings, they will "get all our questions like, "Who's going to the doctor's appointment?' [...] and so we go to the doctor's appointment and ask five questions." Ben also provided an example of a current health question her community has: "we're trying to-- we've heard of PrEP. A lot of people have PrEP, but we don't even know how old you have to be to be on PrEP [...] it literally doesn't say on the website."

Contextual conditions relevant to this narrative are identity, education, and age. These conditions shape how Ben's community is treated, what information they can or cannot access, and how often they can access medical professionals based on when their parents, guardians, or other family members make appointments for them. Socioeconomic status and transportation serve as other contextual conditions since Ben's narrative suggests that at least some community members can afford a doctor's visit and get to the doctor's office.

Education constitutes a significant barrier to Ben and her community, who face homophobia and transphobia in school. Ben recalled a specific negative experience where these barriers intersected:

I have been called a [homophobic slur] in front of my teacher, and the teacher didn't do anything. She just was looking and was literally just texting on her phone. I sit two seats away from her. It's a row. She's in the corner. I was in the row that was three seats. I sit in the back one near the wall, and the boy was next to her desk. And he went right up to my desk and said it to me. And she was just on her phone texting still.

In this account, Ben signifies that school is a violent place for her and the community. Said violence can lead to the invisibility of LGBTQ+ identities illustrated by Ben's teacher ignoring his identity by allowing a peer to demean it. This invisibility is reinforced by the administration, namely the school principal who remains "really silent" on LGBTQ+ issues. While unstated by Ben, the contextual conditions of geography and law, and their related environmental and legal barriers can also produce this invisibility; SC has "no promo homo" laws, which render it illegal for health and sexuality education teachers to discuss homosexuality in a positive light.

A final barrier shaping Ben's community relates to family, as Ben characterized some of her members' families as "broken." This descriptor relates to their lack of acceptance for their LGBTQ+ children, as Ben recounted one community member who "when he first came out to his parents, he had an argument with his family, and they almost told him to get out." The concordant risk of being kicked out of one's home likely shapes how much health information Ben's community can create, seek, share, and use without family becoming aware of their LGBTQ+ identities. 
These contextual conditions and barriers contribute to an information vacuum that produces an immediate lack of information about LGBTQ + health issues, such as information to address the community's PrEP-related question. To defend against these barriers, the community relies on its members to engage in a specific form of community defensive information seeking and sharing in which one member serves as an information intermediary between a medical professional and the community's collective health questions and concerns. Community members employ these community defensive practices to maintain insularity of health questions and concerns based on a keen awareness that adults in their everyday lives do not have the information they need and are either indifferent or antagonistic to their LGBTQ+ identities.

Narrative 3: Shannon. College student Shannon recalled a traumatic experience consequential to her physical and mental health:

I have been gay-bashed. [My partner and I] foolishly thought it was a good idea to go to this-- she loved country music, and we'd go to this country honky-tonk here in [CITY]. It's closed down now. It's a bar called [CLUB]. And we got cornered there in the parking lot afterwards. And it's a pretty scary situation like that. But after surviving that, I really became a staunch advocate of seeking out communities of people like myself because there are safety in numbers.

Shannon's experience of being gay-bashed illustrates the prevalence of physical violence, a significant health issue, experienced by participants and their communities. An essential set of contextual conditions and barriers producing this issue are temporal, as the violence occurred in 2004, which notes a marked difference in public visibility and legal acceptance for almost any LGBTQ+ person in the U.S. Shannon's experience occurred just over a decade before the Obergefell vs. Hodges ruling, which legalized same-sex marriage, and the Equal Rights Employment Commission ruled to include sexual orientation discrimination as a form of illegal discrimination.

Simply because this event happened in the past does not displace its import or likelihood of happening again. This observation is due to other, intersecting contextual conditions including gender identity and sexuality as they pertain to Shannon's understanding of living in a Southern rural town with a lack of legal protections for and political actors supportive of LGBTQ+ communities. Shannon envisions these intersecting conditions as producing legal and ideological barriers, which are unique and still relevant to the state in 2019: "[SC] is notoriously very right-wing. There's not a shred of protection for LGBTQ people at the state level, and there won't be as long as somebody like Henry McMaster is governor of South Carolina." This combination of barriers produced an environment in which not only were Shannon and her partner attacked for being in a same-sex relationship but also had no legal recourse to address the attack or prevent others from being targeted. Further, these barriers created a specter of potential physical violence, a notable risk that Shannon continues to face.

Shannon's resulting information practices are community protective, self-defensive, and self-protective. Specifically, Shannon engages in community protective sharing when advocating her community to "seek" out LGBTQ+ people to ensure "safety in numbers." Further, by taking her advice, Shannon engages in both self-protective and 
self-defensive information seeking. Her seeking out LGBTQ+ communities is selfprotective because it responds to the risk of physical violence. This practice is also selfdefensive in addressing the unique environmental, ideological, and legal barriers she continues to face. The interrelationship between self-protective and self-defensive is illustrated further by Shannon: "If you live in a state like South Carolina that has no nondiscrimination laws, you have to insulate yourself and find places that protect you from the fact that you have no protection."

Narrative 4: Kim and Mocha. Our final narrative focuses on one interview taking place with two co-leaders of the same LGBTQ+ support group, Kim and Mocha. They reflected on the backlash they were receiving at the time of the interview due to Mocha's participation in an upcoming LGBTQ+ themed event at their local library. Upon the event's announcement, their organization's social media sites received threats of physical violence and detractors planned a day-of protest. As Mocha stated: "I didn't think 7,000 people in [CITY] had library cards until they heard [we] were coming to the library." Based on these threats and negative feedback, Kim stated that the upcoming event "has got me just freaked out." She expressed particular concern of physical violence during the event: "I mean, every time I think about it, I think about Mocha getting hurt. And the [other members] getting hurt." As a result of both preexisting threats and the imagined possibilities of what could occur, Kim detailed several strategies for assuring the safety of Mocha and other community members on the day of the event. One such concern for the event was parking that would allow Kim to escort participants to the library. She needed to have a defined location "to put [her] people's cars," ensuring it was the "safest location" in the area. Further, Kim wanted to ensure that any individuals counter-protesting (i.e., supporting the event) "were family" (i.e., belonged to the LGBTQ+ community) and would not be violent toward Mocha or others. Kim laid out potential strategies for action if someone were to try and attack Mocha: "If anybody goes near her, I'll knock [Mocha's partner] to get to her."

Aside from consistent, contextual conditions of gender identity and sexuality, other relevant ones are geography and ideology. These conditions produced related environmental, ideological, homophobia, and transphobia barriers wherein Kim and Mocha's relationship with Southern culture and its ideologies led them to feel unwelcome in multiple institutional spaces, including the library system hosting the event. Spirituality and religion each respectively served as a contextual condition and barrier, as Kim and Mocha both noted that the threats and antagonism came from extremist Christian organizations espousing homophobia and transphobia. Like Shannon, Kim and Mocha also experienced a temporal barrier, noting a resurgence of anti-LGBTQ+ sentiment in the wake of Donald Trump becoming president. Also similar to Shannon, a significant risk identified by Kim and Mocha was physical violence. Moreover, a litany of concerns about the event expressed by Kim and Mocha highlight another risk, fear of the unknown, wherein so many exterior factors could go wrong during the event that they could not possibly anticipate every threat. As Mocha explained: "You just don't know how they're going to come at you." 
In response to these conditions, barriers, and risks, Kim and Mocha engaged in community protective information practices. Specifically, they used seeking to inventory the logistical steps they would need to protect themselves from a litany of risks (e.g., looking for safe places to park) and then created a series of responses to anticipated events (e.g., what to do if someone were to attack). Finally, they engaged in use through the proposed assessment of counter-protestors to determine whether they "were family." While Kim and Mocha did not specify the details of how to make this assessment, they are likely rooted in LGBTQ+ culture and community dynamics, particularly the importance of chosen family among those who may not be accepted for their identities by blood relatives.

\section{Discussion}

Findings continue to advance an information practices approach and information marginalization theory as viable frames from which to identify new dimensions of people's information interactions. Several findings aligned with those of prior theory and literature including the importance of mediation between the community, individual members, and outside information sources; the use of tactical and agentic information practices in response to social and structural barriers, including lack of representation within institutional contexts; and the viability of community as a context from which to assess information practices [22, 25-27, 29]. A significant contribution is the distinction between protective and defensive, extending the work of information marginalization research [22-23]. This distinction highlights the fact that even if a participant does not face immediate constraints to their health information practices (barriers), they can still respond to perceived adverse outcomes produced by these barriers (risks). This finding aligns with a central claim of minority stress theory, positing that absent of immediate barriers, LGBTQ+ people still may experience stressors due to expectations that they will face these barriers in the future [47]. Findings also advance population health [20] and resilience [21] approaches within an informational context by reframing health information practices from resultant of personal failings to empowered and intentional acts. While some of the information practices of participants and communities appear to be uninformed or unsafe, when contextualized within the conceptual model, these practices become well-assessed and vetted within the community.

Findings have implications for service to LGBTQ+ populations within medical institutions. Specifically, we found that communities engage in defensive and protective practices to integrate outside health information into their communities in ways that are socially and informationally relevant. Leaders play a significant role in facilitating these practices by encouraging collective information sharing and exchange and acting as an intermediary between experts and their community. In this way, participants' information practices parallel those adopted by community health workers (CHW) who are trusted members or have specialized knowledge of their community and serve as an intermediary between them and health practitioners. Findings from this research can inform LGBTQ+ CHW training by incorporating the informational elements of health identified by the study's conceptual model. This implication is particularly salient given 
that provisions of the Affordable Care Act coupled with grant funding have increased the number and use of CHWs, however, they continue to lack standardized training [48].

Another practice-oriented implication is for medical professionals to center the nonprofessional knowledge and information practices of communities as central to informing practice. Another participant, Second, illustrates this strategy when explaining why she likes her physician: "She'll ask me for resources, and I'll share what I have, and she'll be like, 'Hey, I read this ... Is this true?' Because sometimes they're given stuff that's not necessarily popular [within the community]." In this interaction, Second's doctor returns some of the power inherent to a patient-expert interaction to Second by seeking information that reflects the values of Second's community. This practice can be facilitated by LIS researchers and practitioners, who through sociocultural, empirical observation of $\mathrm{LGBTQ}^{+}$communities can contribute to continuing education training specifically rooted in cultural competency for medical professionals. These trainings are necessary not only to inform a patient-expert interaction but also to assist medical professionals in sharing information sought and disseminated by a community. Given the consistent practices of mediating and exchanging information between an $\mathrm{LGBTQ}^{+}$member to and for their broader community, we argue that the interoperability of self and community has significant impact in terms of resource and information distribution. While one practitioner may see providing additional information to one $\mathrm{LGBTQ}^{+}$person as time-consuming or ineffective, the findings suggest that the extended distribution of this information has a ripple effect and can aid countless community members in unseen and unknown ways.

While this study has attended to clear gaps in representation across age, race/ethnicity, class, and regional lines, many groups remain overlooked. Particular absences existed among Latinx communities, a notable presence in SC. Moreover, while participants represented diverse identities within the broad LGBTQ+ umbrella, we were unable to represent specific identities, e.g., two-spirit, genderfluid, demisexual. Further, we were limited by which SC LGBTQ+ communities wanted to be visible, as some remain purposefully hidden. We had a particular lack of participant representation from Charleston, which was somewhat surprising to us given the visibility and reach of prominent LGBTQ+ organizations in this area. Finally, we did not represent particular professions whose LGBTQ+ members face unique health needs, such as sex workers. The collectively missed populations noted here mean that our study overlooked multiple health issues and concerns.

We also acknowledge the inherent epistemic violence [49] latent to the categorical work done in our coding, naming, and mapping of LGBTQ+ information practices. Our choice to deploy ambit terms such as community/self and defensive/protective information practices reinforce the very binaries that LGBTQ+ individuals unsettle; in fact, one could describe many of our participants as existing within and doing these practices simultaneously. Further, as LGBTQ+ individuals, our participants' orientation toward concepts like community and self assume a universalized notion of these concepts rather than something experienced as a point of disorientation or even multiplicity [50]. Therefore, while we did not aim to essentialize our participants' 
experiences, the very act of naming these categories occurred and subsequent essentialisms emerged.

\section{Conclusion}

This research examined the health information practices of SC LGBTQ+ communities, addressing how sociocultural context shapes these practices. Informed by thirty semistructured interviews with community leaders, we developed a conceptual framework, which uncovered two distinct types of information practices, protective and defensive. This framework advances sociocultural theories of information practices, providing a preliminary step to theory-building and contributes to research extending our conceptual model to other groups experiencing information marginalization. Findings also offer ways for medical professionals to improve service to LGBTQ+ populations.

This work has several future directions: focus groups with community members to validate and expand findings from leader interviews; employment of more nuanced and embedded strategies to recruit participants underrepresented in the current sample; enhanced analysis of information worlds maps based on recent analytical recommendations from Greyson, Stoeveller, \& Shankar (2019); and interviews with health and medical librarians, as well as medical practitioners to understand their information practices in relation to the LGBTQ+ communities they serve. Ultimately, this study and the future work discussed seeks to alter current perceptions of SC LGBTQ+ communities' health information practices as being inherently lacking to being purposeful and agentic. In doing so, our findings can inform both theory and praxis in generative directions by illuminating alternative ways of imagining not only how LGBTQ+ communities tactically deal with health information, but also how medical and information institutions can value LGBTQ + communities as an already information-rich resource.

\section{References}

1. Institute of Medicine. The health of lesbian, gay, bisexual, and transgender people: Building a foundation for better understanding. National Academic Press, Washington, D.C. (2011).

2. Center for Disease Control and Prevention, LGBT Youth Homepage, https://www.cdc.gov/lgbthealth/youth.htm, last accessed 2019/11/19.

3. HealthyPeople.gov Lesbian, Gay, Bisexual, and Transgender Health U.S. Department of Health Evidence-based Resources Homepage, https://www.healthypeople.gov/2020/topicsobjectives/topic/lesbian-gay-bisexual-andtransgender-health/ebrs, last accessed 2019/11/19.

4. James, S.E., Herman, J. L., Rankin, S., Keisling, M., Mottet, L., \& Anaf, M. The report of the 2015 U.S.C transgender survey. National Center for Transgender Equality, Washington, D.C. (2016). $\quad \mathrm{https}: / /$ www.transequality.org/sites/default/files/docs/usts/USTS\%20Full\%20Report\%20-\%20FINAL\%201.6.17.pdf, last accessed 2019/11/19. 
5. Transgender Law Center at Southerners on New Ground. The Grapevine: A Southern Trans Report: Prioritizing Issues Impacting Transgender, Gender Nonbinary, and Gender Nonconforming Southerners. (2019). http://transgenderlawcenter.org/wpcontent/uploads/2019/05/grapevine_report_eng-FINAL.pdf, last accessed 2019/11/19.

6. Morris, M., \& Hawkins, B. Towards a new specialization in health librarianship: LGBTQ health. Journal of the Canadian Health Libraries Association, 37(1), 20-23 (2016). https://doi.org/10.5596/c16-007.

7. Romanelli, M., \& Hudson, K. D. Individual and systemic barriers to health care: Perspectives of lesbian, gay, bisexual, and transgender adults. American Journal of Orthopsychiatry, 87(6), 714-728 (2017). https://doi.org/10.1037/ort0000306.

8. Perrin, P. B., Sutter, M. E., Trujillo, M. A., Henry, R. S., \& Pugh Jr, M. The minority strengths model: Development and initial path analytic validation in racially/ethnically diverse LGBTQ individuals. Journal of Clinical Psychology, 1-19 (2019). https://doi.org/10.1002/jclp.22850.

9. Matthews, D. D., \& Lee, J. G. A profile of North Carolina lesbian, gay, and bisexual health disparities, 2011. American Journal of Public Health, 104(6), 98-105 (2014). https://doi.org/ 10.2105/AJPH.2013.301751.

10. Williams Institute. LGBT people in South Carolina (2018). https://williamsinstitute.law.ucla.edu/wp-content/uploads/South-Carolina-fact-sheet.pdf, last accessed 2019/11/19.

11. Mallory, C. \& Sears, B. Discrimination against LGBT people in South Carolina. UCLA School of Law Williams Institute, Los Angeles, CA (2019). https://williamsinstitute.law.ucla.edu/wp-content/uploads/South-Carolina-ND-July2019.pdf, last accessed 2019/11/19.

12. Coleman, J. D., Irwin, J. A., Wilson, R. C., \& Miller, H. C. The South Carolina LGBT needs assessment: A descriptive overview. Journal of Homosexuality, 61(8), 1152-1171 (2014). https://doi.org/10.1080/00918369.2014.872515.

13. Savolainen, R. Information behavior and information practice: reviewing the "umbrella concepts" of information-seeking studies. The Library Quarterly, 77(2), 109-132 (2007). https://www.jstor.org/stable/10.1086/517840.

14. Savolainen, R. Everyday information practices: a social phenomenological perspective. Scarecrow Press, Lanham, MD (2008).

15. McKenzie, P. J. A model of information practices in accounts of everyday-life information seeking. Journal of Documentation, 59(1), 19-40 (2003). https://doi.org/10.1108/00220410310457993.

16. Frohmann, B. (1992). The power of images: A discourse analysis of the cognitive viewpoint. Journal of Documentation, 48(4), 365-386 (1992). https://doi.org/10.1108/eb026904.

17. Talja, S. Constituting "information" and "user" as research objects: a theory of knowledge formations as an alternative to the information-man theory. In: Vakkari, P., Savolainen, R., \& Dervin, B. (eds). INFORMATION SEEKING IN CONTEXT: PROCEEDINGS OF AN INTERNATIONAL CONFERENCE ON RESEARCH IN INFORMATION NEEDS, SEEKING AND USE IN DIFFERENT CONTEXTS, pp. 67-80. London: Taylor Graham (1997).

18. Julien, H. Where to from here? Results of an empirical study and user-centered implications for information design. In: Wilson, T.D., and Allen, D.K. (eds.). Exploring the Contexts of Information Behaviour, (pp. 586-596) Taylor Graham, London, UK (1999).

19. Savolainen, R. Everyday life information seeking: Approaching information seeking in the context of "way of life." Library \& Information Science Research, 17(3), 259-294 (1995). https://doi.org/10.1016/0740-8188(95)90048-9. 
20. Marmot, M. \& Wilkinson, R. Social Determinants of Health. Oxford University Press, New York, NY (2005).

21. Colpitts, E., \& Gahagan, J. The utility of resilience as a conceptual framework for understanding and measuring LGBTQ health. International Journal for Equity in Health, 15(1), 60 (2016). https://doi.org/10.1186/s12939-016-0349-1.

22. Gibson, A. N., \& Martin III, J. D. Re-situating information poverty: Information marginalization and parents of individuals with disabilities. Journal of the Association for Information Science and Technology, 70(5), 476-487 (2019). https://doi.org/10.1002/asi.24128.

23. Chatman, E. A. The impoverished life-world of outsiders. Journal of the American Society for Information Science, 47(3), 193-206 (1996). https://doi.org/10.1002/(SICI)10974571(199603)47:3\%3C193::AID-ASI3\%3E3.0.CO;2-T.

24. Thompson, K. M. Furthering understanding of information literacy through the social study of information poverty. The Canadian Journal of Information and Library Science, 31(1), 87115 (2007).

25. Lingel, J. Information tactics of immigrants in urban environments. Information Research, 16(4) (2011). http://www.informationr.net/ir/16-4/paper500.html, last accessed 2019/11/16.

26. Greyson, D. Health information practices of young parents. Journal of Documentation, 73(5), 778-802 (2017). https://doi.org/10/1108/JD-07-2016-0089.

27. Veinot, T. C. A multilevel model of HIV/AIDS information/help network development. $\begin{array}{llll}\text { Journal of } & \text { Documentation, 66(6), }\end{array}$ https://doi.org/10.1108/00220411011087850.

28. Kitzie, V. "That looks like me or something I can do": Affordances and constraints in the online identity work of US LGBTQ+ millennials. Journal of the Association for Information Science and Technology, 70(12) 1340-1351 (2019). https://doi.org/10.1002/asi.24217.

29. Floegel, D., \& Costello, K. L. Entertainment media and the information practices of queer individuals. Library \& Information Science Research, 41(1), 31-38 (2019). https://doi.org/10.1016/j.lisr.2019.01.001.

30. Steinke, J., Root-Bowman, M., Estabrook, S., Levine, D. S., \& Kantor, L. M. Meeting the needs of sexual and gender minority youth: formative research on potential digital health interventions. Journal of Adolescent Health, 60(5), 541-548 (2017). https://doi.org/10.1016/j.jadohealth.2016.11.023.

31. Schmitz, R. M., Sanchez, J., \& Lopez, B. LGBTQ+ Latinx young adults' health autonomy in resisting cultural stigma. Culture, Health \& Sexuality, 21(1), 16-30 (2019). https://doi.org/10.1080/13691058.2018.1441443.

32. Duggan, L. (2012). The twilight of equality?: Neoliberalism, cultural politics, and the attack on democracy. Beacon Press, Boston, MA (2012).

33. Spade, D. Normal life: Administrative violence, critical trans politics, and the limits of law. Southend Press, New York, NY (2015).

34. Puar, J. K., \& Rai, A. Monster, terrorist, fag: The war on terrorism and the production of docile patriots. Social Text, 20(3), 117-148 (2002). https://www.muse.jhu.edu/article/31948, last accessed 2019/11/19.

35. Halberstam, J. (2005). In a queer time and place: Transgender bodies, subcultural lives. New York University Press, New York, NY (2005).

36. Strauss, A., \& Corbin, J. Basics of qualitative research techniques. SAGE, Thousand Oaks, CA (1998).

37. Saldaña, J. The coding manual for qualitative researchers. SAGE, Thousand Oaks, CA (2015).

38. Hillary, G. A. Definitions of community: Areas of agreement. Rural Sociology, 20, 111-123 (1995). 
39. Worthen, M. G. The interactive impacts of high school gay-straight alliances (GSAs) on college student attitudes toward LGBT individuals: An investigation of high school characteristics. Journal of Homosexuality, 61(2), 217-250 (2014). https://doi.org/10.1080/00918369.2013.839906.

40. Weiser, S. M. The art of resistance: An arts based understanding of activism. Unpublished doctoral dissertation, Columbia, SC (2018). https://scholarcommons.sc.edu/etd/4777, last accessed 2019/11/19.

41. Koh, K. Adolescents' information-creating behavior embedded in digital $\mathrm{M}$ edia practice using scratch. Journal of the American Society for Information Science and Technology, 64(9), 1826-1841 (2013). https://doi.org/10.1002/asi.22878.

42. Greyson, D. Information world mapping: A participatory, visual, elicitation activity for information practice interviews. In: Grove, A. (ed.) PROCEEDINGS OF THE AMERICAN SOCIETY FOR INFORMATION SCIENCE AND TECHNOLOGY, vol. 50, issue 1, pp. 14 (2013).

43. Strauss, A., \& Corbin. J. Grounded theory methodology: An overview. In: Denzin, N. and Lincoln, Y. (eds). Handbook of Qualitative Research (pp. 273-85). Thousand Oaks, CA: SAGE, Thousand Oaks, CA (1994).

44. Charmaz, K. Constructing grounded theory. SAGE, Thousand Oaks, CA (2014).

45. Creswell, J.W. Qualitative inquiry \& research design: Choosing among five approaches. SAGE, Los Angeles, CA (2013).

46. Geertz, C. The interpretation of cultures. Basic Books, New York, NY (1973).

47. Dentato, M. P., Halkitis, P. N., \& Orwat, J. Minority stress theory: An examination of factors surrounding sexual risk behavior among gay and bisexual men who use club drugs. Journal of Gay \& Lesbian Social Services, 25(4), 509-525 (2013). https://doi.org/10.1080/10538720.2013.829395.

48. Kangovi, S., Grande, D., \& Trinh-Shevrin, C. From rhetoric to reality-community health workers in post-reform US health care. The New England Journal of Medicine, 372(24), 2277 (2015). https://doi.org/10.1056/NEJMp1502569.

49. Spivak, G. G. "Can the subaltern speak?" In: Nelson, C. and Grossberg, L. (eds.) Marxism and Interpretation of Culture (pp. 66-111). University of Illinois Press, Urbana, IL (1988).

50. Ahmed, S. Queer phenomenology: Orientations, objects, others. Duke University Press, Durham, NC (2006). 